Technology and Software DeVelopment

A New Methodology to Analyze Instabilities in SEM 4 Imaging

Catalina Mansilla, Václav Ocelík, and Jeff T. M. De Hosson

Convenient Preparation of High Quality Specimens for Annealing Experiments in the

Transmission Electron Microscope

Martial Duchamp, Qiang Xu, and Rafal Dunin-Borkowski

A Multi-Step Transmission Electron Microscopy Sample Preparation Technique for Cracked, Heavily Damaged, Brittle Materials

Claire V. Weiss Brennan, Scott D. Walck, and Jeffrey J. Swab

Replica Extraction Method on Nanostructured Gold Coatings and Orientation Determination Combining SEM and TEM Techniques

Christian Bocker, Michael Kracker, and Christian Rüssel

The Maximum Separation Cluster Analysis Algorithm for Atom-Probe Tomography: Parameter Determination and Accuracy

Eric Aimé Jägle, Pyuck-Pa Choi, and Dierk Raabe

Resonant Frequency and Sensitivity of a Caliper Formed With Assembled Cantilever Probes

Based on the Modified Strain Gradient Theory

Mohammad Abbasi and Seyed E. Afkhami

Materials Applications

Spectral Analysis of Irregular Roughness Artifacts Measured by Atomic Force and Laser

Scanning Microscopy

Yuhang Chen, Tingting Luo, Chengfu Ma, Wenhao Huang, and Sitian Gao

Dynamic Modeling and Simulation of Rough Cylindrical Micro/Nanoparticle Manipulation

with Atomic Force Microscopy

Moharam H. Korayem, Hedieh Badkoobeh Hezaveh, and Moein Taheri

The Impact of Lubricants on the Precision Lapping Process

Xionghua Jiang, Zhenxing Chen, Joy Wolfram, Zhongxian Wei, Yuqiu Shen, and Zhizhou Yang

The Influence of Experimental Parameters and Specimen Geometry on the Mass Spectra of

Copper During Pulsed-Laser Atom-Probe Tomography

R. Prakash Kolli and Frederick Meisenkothen

Co-Precipitated and Collocated Carbides and Cu-Rich Precipitates in a Fe-Cu Steel Characterized by Atom-Probe Tomography

R. Prakash Kolli and David N. Seidman

Simultaneous Quantification of Indium and Nitrogen Concentration in InGaNAs Using HAADF-STEM

Tim Grieb, Knut Müller, Emmanuel Cadel, Andreas Beyer, Marco Schowalter, Etienne Talbot, Kerstin Volz, and Andreas Rosenauer

Thickness and Rotational Effects in Simulated HRTEM Images of Graphene on

Hexagonal Boron Nitride Avery J. Green and Alain C. Diebold

Atom Column Indexing: Atomic Resolution Image Analysis through a Matrix Representation Xiahan Sang, Adedapp A. Oni, and James M. LeBeau

Structure Identification in High-Resolution Transmission Electron Microscopy Images:

An Example on Graphene

Jacob S. Vestergaard, Jens Kling, Anders B. Dahl, Thomas W. Hansen, Jakob B. Wagner, and Rasmus Larsen

Chemical Quantification of Atomic-Scale EDS Maps under Thin Specimen Conditions Ping Lu, Eric Romero, Shinbuhm Lee, Judith L. MacManus-Driscoll, and Quanxi Jia Atomic Scale Studies of La/Sr Ordering in Colossal Magnetoresistant $\mathrm{La}_{2}-{ }_{2 \mathrm{x}} \mathrm{Sr}_{1+2 \mathrm{x}} \mathrm{Mn}_{2} \mathrm{O}_{7}$ Single Crystals

Manuel A. Roldan, Mark P. Oxley, Qing'an Li, Hong Zheng, K. E. Gray, J. F. Mitchell, Stephen J. Pennycook, and María Varel

Microstructural Characterization of Dehydrogenated Products of the $\mathrm{LiBH}_{4}-\mathrm{YH}_{3}$ Composite Ji Woo Kim, Kee-Bum Kim, Jae-Hyeok Shim, Young Whan Cho, and Kyu Hwan Oh

Scanning Electron Microscopy and Transmitted Electron Backscatter Diffraction Examination of Asbestos Standard Reference Materials, Amphibole Particles of Differing Morphology, and Particle Phase Discrimination from Talc Ores Bryan R. Bandli and Mickey E. Gunter

In Situ Transmission Electron Microscopy of Ionic Conductivity and Reaction Mechanisms in Ultrathin Solid Oxide Fuel Cells

Amir H. Tavabi, Shigeo Arai, Shunsuke Muto, Takayoshi Tanji, and Rafal E. Dunin-Borkowski

FIB-Etching of Polymer/Metal Laminates and its Effect on Mechanical Performance

E. Faber, W.P. Vellinga, and J.Th.M. De Hosson

Biological Applications

Three-Dimensional Reconstruction of Skeletal Muscle Extracellular Matrix Ultrastructure Allison R. Gillies, Eric A. Bushong, Thomas J. Deerinck, Mark H. Ellisman, and Richard L. Lieber

A Zebrafish Embryo Behaves both as a "Cortical Shell-Liquid Core" Structure and a

Homogeneous Solid when Experiencing Mechanical Forces Fei Liu, Dan Wu, and Ken Chen

Erythrocyte-Platelet Interaction in Uncomplicated Pregnancy

Albe C. Swanepoel and Etheresia Pretorius

Malathion Induced Granulosa Cell Apoptosis in Caprine Antral Follicles: An Ultrastructural and Flow Cytometry Analysis

Jitender Kumar Bhardwaj and Priyanka Saraf

Fine Structure of Neurally Differentiated iPS Cells Generated from a Multiple Sclerosis (MS) Patient:

A Case Study

Daniella Herszfeld, Natalie L. Payne, Aude Silvan, Guizhi Sun, Claude C. Bernard, Joan Clark, and Henry Sathananthan

A New Method for Morphometric Analysis of Opal Phytoliths from Plants

Welmoed A. Out, José F. Pertusa Grau, and Marco Madella

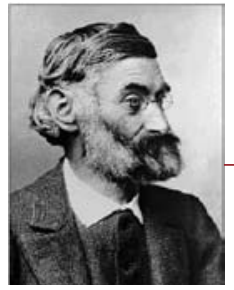

DearAbbe

Dear Abbe,

There was an odd suggestion at our society's executive council meeting. They noted that we needed to create more biological memorial symposia for our meetings. One suggestion was to arrange the death of two prominent biologists. We were dead serious. Can you suggest a good way to accomplish this?

\section{Morbid in Minneapolis}

\section{Dear Morbid,}

I hesitate to even broach this subject since it might implicate me on several mysterious passings of prominent persons from many years ago. Some of them may have even deserved it. Of course, this typically creates some complications and rather lengthy terms of incarceration. But! All is not lost. As any biologist knows, there are different kinds of death. "Brain dead," for instance, where the person is an obvious fool who refuses to understand your brilliant research. What you want is "academic" or "career death," where the scientist is still attending committee meetings and publishing articles but only cites their past research and the accepted theories and does no new work. This is where memorial symposia are especially useful. Holding such a symposium for someone still un-retired is tantamount to career death (like a movie or television actor who now does commercials). After all, if they were still doing anything original, they wouldn't be "honored" by a memorial symposium, nicht wahr? After such an honor"You're done, here's your lifetime achievement award, your office has been moved down the hall with the rest of the emeriti." Be sure to pick someone who keeps writing poor reviews for your articles and grant applications.

\section{Dear Abbe,}

Have you heard the exciting news that Eric Betzig, Stefan Hell, and William Moerner have won the 2014 Nobel Prize for their development of super-resolution fluorescence microscopy? This marks at least the third time that microscopists have been honored for their outstanding contributions to science. Does it ever make you angry that your work was never recognized by the Nobel committee?

\section{Samantha in Stockholm}

\section{Dear Sammie,}

Bedeutungslos! I mean, who cares really? It's not like any of them are going to be invited as guests on the Tonight show. The best they could hope for would be a guest spot on Conan, but seriously, does anyone watch TBS these days? Angry, you ask? Why would I be ANGRY!!!!!

Worried about the effect your discoveries may have on the future of humanity? Can't get that hunky lab manager to have a cup with you after hours? No problem! Send your queries to Herr Abbe via his capable and slightly demented assistant at jpshield@ uga.edu. 